\title{
Decentralized Control with Input Saturation: a First Step Toward Design
}

\author{
Anton A. Stoorvogel [1]
}

Jasmine Minteer [2]

\begin{abstract}
This article summarizes important observations about control of decentralized systems with input saturation and provides a few examples that give insight into the structure of such systems.
\end{abstract}

\section{INTRODUCTION}

Decentralized control systems are characterized by systems that are made up of smaller systems, or agents, which have only indirect information about the other systems. That is, each agent does not receive direct information about the outputs or the inputs to any other agent. Examples of decentralized systems are abundant and include power systems, economic systems, and systems of multiple autonomous agents, to name a few. Control of such systems has been the subject of much research since Wang and Davison's seminal paper [1] in 1973, in which they prove necessary and sufficient conditions for stabilization of linear, timeinvariant (LTI) decentralized systems by LTI controllers.

One of the most important concepts in decentralized control is that of fixed modes, and many different characterizations of fixed modes have been developed over the past three decades (see, for example [2], [3]). The role that fixed modes play in stabilization of decentralized systems has also been studied in detail for both LTI controllers [1] and for linear, time-variant (LTV) controllers [4]. It has been established that LTI controllers can stabilize a decentralized control system if and only if all of the fixed modes are stable. It has also been shown the LTV controllers are capable of moving some (but not all) fixed modes with a precise characterization given in [5].

There are currently many open research areas in decentralized control. One important open problem is stabilization of decentralized systems with input constraints, which we consider in this article.

This article is organized as follows. In Section II, we briefly summarize some important concepts about decentralized control systems that we make use of throughout

\footnotetext{
${ }^{1}$ Department of Electrical Engineering, Mathematics and Computer Science, Delft University of Technology, P.O. Box 5031, 2600 GA Delft, The Netherlands, E-mail: a.a.stoorvogel@ewi.tudelft.nl, Dept. of Mathematics and Computing Science, Eindhoven University of Technology, P.O. Box 513, 5600 MB Eindhoven, The Netherlands. E-mail: A.A.Stoorvogel@tue.nl.

${ }^{2}$ School of Electrical Engineering and Computer Science, Washington State University, Pullman, WA 99164-2752, U.S.A. E-mail: jminteer@eecs.wsu.edu.

${ }^{3}$ Department of Electrical Engineering, Mathematics and Computer Science, Delft University of Technology, P.O. Box 5031, 2600 GA Delft, The Netherlands, E-mail: c.deliu@ewi.tudelft.nl
}

the rest of the paper. In Section III, we investigate the ability of nonlinear, time-invariant controllers to move fixed modes at zero. We show, through an example, that applying such controllers results in multiple equilibria; thus, they cannot be used (at least under some conditions) to stabilize a system with a fixed mode at zero. In Section IV, we demonstrate the design of a completely linear observerbased full-state feedback controller for a system with zero eigenvalues that are not simple after preliminary feedback is applied. This example illustrates some of the problems that can arise in stabilizing a simple decentralized system that would not exist if the system were centralized. Finally, in Section $\mathrm{V}$, we introduce a method for model reduction by singular perturbation that can be a useful first step in controller design. Not surprisingly, the decentralized structure adds some complexity to the process of model reduction, which we explore in detail.

\section{Decentralized Structure and Fixed Modes}

A decentralized control system has the following structure:

$$
\Sigma:=\left\{\begin{array}{l}
\dot{x}=A x+\sum_{1}^{k} B_{i} u_{i} \\
y_{i}=C_{i} x, \quad i=1, \ldots, k
\end{array}\right.
$$

where $A \in \mathbf{R}^{n \times n}, B_{i} \in \mathbf{R}^{n \times m_{i}}, C_{i} \in \mathbf{R}^{p_{i} \times n}, m_{i}$ is the number of inputs to the $i^{t h}$ agent, and $p_{i}$ is the number of measurements available to the $i^{t h}$ agent.

As mentioned, one of the most important concepts in decentralized control theory is that of fixed modes. As defined in [1], a fixed mode is any mode that cannot be moved by static or dynamic LTI feedback. That is, if $K_{i} \in \mathbf{R}^{m_{i} \times p_{i}}$ is the feedback to the $i^{t h}$ agent, then a fixed mode is any mode that is an eigenvalue of

$$
A+\sum_{i=1}^{v} B_{i} K_{i} C_{i}
$$

for all $K_{i}$. Wang and Davison also show in [1] that if a mode is a fixed mode under static LTI feedback, then it is also a fixed mode under dynamic LTI feedback. However, in the case of input constraints, the controllers are, in general, nonlinear; therefore, the results regarding fixed modes do not immediately apply. However, it can be easily shown that if we require local exponential stability, then fixed modes must be in the open left half plane, or exponential stability cannot be attained. 


\section{NONLINEAR CONTROLLERS AND FIXED MODES AT} ZERO

Although it has been shown in [5]-[7] that linear, timevarying controllers can move some fixed modes, the same is not true in general for nonlinear, time-invariant controllers. Gong and Aldeen showed in [5] that the so-called quotient fixed modes cannot be moved by any nonlinear, time-varying controller. However, an interesting question is whether time-varying controllers are needed, and in this paper we will give an example of a system with a fixed mode at the origin that is not a quotient fixed mode but cannot be moved by any nonlinear, time-invariant controller. Specifically, we consider dynamic, time-invariant, nonlinear controllers of the form

$$
\Sigma_{c_{i}}=\left\{\begin{array}{l}
\dot{v}_{i}(t)=g_{i}\left(y_{i}(t), v_{i}(t)\right), \quad v_{i}\left(t_{0}\right)=v_{i, 0} \\
u_{i}(t)=f_{i}\left(y_{i}(t), v_{i}(t)\right)
\end{array}\right.
$$

where $g_{i}(\cdot)$ and $f_{i}(\cdot)$ are any nonlinear, time-invariant functions of the $i^{\text {th }}$ controller. The results to follow also apply to systems with static, nonlinear controllers, since static controllers are just a special case of the controllers described in (2). Since we are trying to make the origin an asymptotically stable equilibrium, without loss of generality, we assume that $g_{i}(0,0)=0$ and $f_{i}(0,0)=0$.

We will show that applying controllers of this form to a system with a fixed mode at zero will often result in a continuum of multiple equilibria. This will be illustrated by the following example.

Example 1 Consider the system

$$
\Sigma_{1}:=\left\{\begin{array}{l}
\dot{x}=A x+\sum_{1}^{2} B_{i} u_{i} \\
y_{i}=C_{i} x, \quad i=1,2
\end{array}\right.
$$

where

$$
\begin{gathered}
A=\left(\begin{array}{ccc}
-1 & 1 & 1 \\
1 & 0 & 0 \\
-2 & 0 & 0
\end{array}\right), \quad B_{1}=\left(\begin{array}{l}
1 \\
0 \\
0
\end{array}\right), \quad B_{2}=\left(\begin{array}{l}
0 \\
0 \\
1
\end{array}\right) \\
C_{1}=\left(\begin{array}{lll}
0 & 1 & 0
\end{array}\right), \quad C_{2}=\left(\begin{array}{lll}
1 & 0 & 0
\end{array}\right) .
\end{gathered}
$$

This system is centralized controllable and observable and has the following eigenvalues:

$$
\left\{\lambda_{1}, \lambda_{2}, \lambda_{3}\right\}=\left\{0,-\frac{1}{2} \pm i \frac{\sqrt{3}}{2}\right\} .
$$

Also, by the characterization in [2], [3], $\lambda_{1}=0$ is a fixed mode, since

$$
\operatorname{rank}\left(\begin{array}{cc}
A & B_{1} \\
C_{2} & 0
\end{array}\right)=\operatorname{rank}\left(\begin{array}{cccc}
-1 & 1 & 1 & 1 \\
1 & 0 & 0 & 0 \\
-2 & 0 & 0 & 0 \\
1 & 0 & 0 & 0
\end{array}\right)=2 .
$$

Since this system is strongly connected (that is there exists a path in the system from control station one to control station two and vice versa) and centralized controllable and observable, it has no quotient fixed modes. When we apply static controllers $u_{1}=f_{1}\left(x_{2}\right)$ and $u_{2}=f_{2}\left(x_{1}\right)$ to the first two control stations, then we have multiple equilibria characterized by:

$$
\begin{aligned}
& x_{1}(t)=0 \\
& x_{2}(t)=\alpha \\
& x_{3}(t)=-\alpha-f_{1}(\alpha)
\end{aligned}
$$

If we apply dynamic feedback of the form (2) to the first two control stations, then applying these controllers is equivalent to applying the following static controllers:

$$
\tilde{u}_{1}=\left(\begin{array}{l}
f_{1}\left(\tilde{y}_{1}\right) \\
g_{1}\left(\tilde{y}_{1}\right)
\end{array}\right) \quad \tilde{u}_{2}=\left(\begin{array}{l}
f_{2}\left(\tilde{y}_{2}\right) \\
g_{2}\left(\tilde{y}_{2}\right)
\end{array}\right)
$$

to the system:

$$
\tilde{\Sigma}_{1}:=\left\{\begin{array}{l}
\dot{\tilde{x}}=\tilde{A} \tilde{x}+\sum_{1}^{2} \tilde{B}_{i} \tilde{u}_{i} \\
\tilde{y}_{i}=\tilde{C}_{i} \tilde{x}, \quad i=1,2
\end{array}\right.
$$

where

$$
\begin{gathered}
\tilde{A}=\left(\begin{array}{ccc}
A & 0 & 0 \\
0 & 0 & 0 \\
0 & 0 & 0
\end{array}\right), \quad \tilde{B}_{1}=\left(\begin{array}{cc}
B_{1} & 0 \\
0 & I \\
0 & 0
\end{array}\right), \quad \tilde{B}_{2}=\left(\begin{array}{cc}
B_{2} & 0 \\
0 & 0 \\
0 & I
\end{array}\right) \\
\tilde{C}_{1}=\left(\begin{array}{ccc}
C_{1} & 0 & 0 \\
0 & I & 0
\end{array}\right), \quad \tilde{C}_{2}=\left(\begin{array}{ccc}
C_{2} & 0 & 0 \\
0 & 0 & I
\end{array}\right) .
\end{gathered}
$$

If we look for equilibria, then we find $v_{2}(t)=0$, and for any given function $f_{1}$, we need that

$$
\begin{aligned}
& x_{1}(t)=0 \\
& x_{2}(t)=\alpha \\
& x_{3}(t)=-\alpha-f_{1}\left(\alpha, v_{1,0}\right) .
\end{aligned}
$$

Finally, we note that we need $g_{1}\left(\alpha, v_{1,0}\right)=0$. Note that if the controller is dynamic of order 1 , then $v_{1}$ is scalar valued. In that case, $g_{1}\left(\alpha, v_{1,0}\right)=0$ has either an infinite number of zeros close to the origin or an extremum. In the first case, we have an infinite number of equilibria, while in the second case, it is easy to check that the system cannot be asymptotically stable. Although we do not have a full proof yet, we conjecture that also higher-order timeinvariant controllers cannot stabilize this system.

\section{Single Integrator ExAmple}

In this section, we design and simulate a controller for a decentralized, single-integrator system with input constraints. We will show some of the inherent limitations imposed both by the decentralized structure and the input constraints.

Consider the following system:

$$
\Sigma_{2}:=\left\{\begin{array}{l}
\dot{x}=A x+\sum_{1}^{3} B_{i} u_{i} \\
y_{i}=C_{i} x, \quad i=1,2,3
\end{array}\right.
$$


where

$$
\begin{gathered}
A=\left(\begin{array}{lll}
0 & 0 & 0 \\
0 & 0 & 0 \\
0 & 0 & 0
\end{array}\right), \quad B_{1}=\left(\begin{array}{l}
1 \\
0 \\
0
\end{array}\right), \\
B_{2}=\left(\begin{array}{l}
0 \\
1 \\
0
\end{array}\right), \quad B_{3}=\left(\begin{array}{l}
0 \\
0 \\
1
\end{array}\right), \\
C_{1}=\left(\begin{array}{lll}
0 & 1 & 0
\end{array}\right), \quad C_{2}=\left(\begin{array}{lll}
0 & 0 & 1
\end{array}\right), \\
C_{3}=\left(\begin{array}{lll}
1 & 0 & 0
\end{array}\right),
\end{gathered}
$$

where $\sigma(u)$ is the standard saturation function given by

$$
\sigma(u):= \begin{cases}-1, & u<-1 \\ u, & |u| \leq 1 \\ 1, & u>1\end{cases}
$$

If this system were centralized, then applying static, linear feedback of the form

$$
u=-\epsilon C^{-1} y
$$

(where $\epsilon$ is an arbitrary positive constant) will achieve global stability, as long as $\epsilon$ is small enough so that the inputs never saturate. However, since the system has a decentralized structure, global stabilization is not achievable with linear control.

After applying linear controllers to the first two channels, the system is locally linear and still has three zero eigenvalues. This implies that the linear system, which must be stabilizable through the third (scalar) input, cannot be stable and actually has a Jordan block of size three. The results from [8], [9] show that global stabilization of this linear system then requires nonlinear controllers. However, after applying the first two controllers, the saturation elements become state constraints that limit the growth rate of the state to be linear; hence we might potentially be able to achieve global stability by linear controllers. But it requires making explicit use of the saturation elements in this system, which is obviously highly complex.

However, semi-global stabilization is achievable and is much easier to obtain. By applying static feedback to the first two agents (which we refer to as preliminary feedback), the system can be made both observable and controllable by the third agent. (See [10].) Thus, we can apply an observerbased, full-state feedback controller at the third agent.

In typical design procedures, the controller is designed to meet certain criteria, and then the observer is designed so that the estimated state converges "quickly" to the true state. However, to date, no real design methods have been developed for decentralized systems. Our objective in this example is to stabilize the system without saturating any of the inputs (thus keeping the system totally linear), or at least to design a stabilizing controller that only saturates for a very short period of time. The procedure we follow is to first design the observer and then to design the state feedbacks by a low-gain design method (see [11]) so that the inputs do not saturate. It should be noted that our design involved significant trial and error and that developing controller design methods for decentralized systems continues to be an open research area.

Preliminary Feedback We apply the following preliminary feedbacks to make the system observable and controllable from the third agent: $u_{1}=k_{1} y_{1}=k_{1} x_{2}$ and $u_{2}=k_{2} y_{2}=k_{2} x_{3}$. If neither of these inputs saturates, then after applying these preliminary feedbacks, the system as viewed from station three is:

$$
\begin{aligned}
& \dot{x}=\underbrace{\left(\begin{array}{ccc}
0 & k_{1} & 0 \\
0 & 0 & k_{2} \\
0 & 0 & 0
\end{array}\right)}_{\tilde{A}} x+B_{3} \sigma\left(u_{3}\right) \\
& y_{3}=C_{3} x .
\end{aligned}
$$

Clearly, this system is both controllable and observable $\forall k_{1}, k_{2} \neq 0$

Observer and Controller Design We now design a linear observer at station three and then a full-state feedback controller using a low-gain design method (see [11]) so that $\left\|u_{3}(t)\right\| \leq 1 \forall t \geq T$, where $T$ can be chosen to be arbitrarily small.

The observer at station three is described by the following equation:

$$
\dot{\hat{x}}=\tilde{A} \hat{x}+B_{3} \sigma\left(u_{3}\right)+L\left(y_{3}-C_{3} \hat{x}\right),
$$

and the observer error, $\delta=\hat{x}-x$, has the following dynamics:

$$
\dot{\delta}=\dot{\hat{x}}-\dot{x}=\left(\tilde{A}-L C_{3}\right) \delta
$$

Since $\left(\tilde{A}, C_{3}\right)$ is observable, the eigenvalues of $\tilde{A}-$ $L C_{3}$ can be placed in any desired location. In this example, $L$ was designed by placing the eigenvalues of $\tilde{A}-L C_{3}$ at $\{-0.1,-0.11,-0.12\}$. These locations were arbitrarily chosen, since the only requirement on the observer dynamics is that the error decays exponentially to zero. The resulting observer gain matrix was $L^{\prime}=$ $\left(\begin{array}{lll}0.3300 & 0.3620 & 0.1320\end{array}\right)$.

The controller at station three has the following form:

$$
u_{3}=-F_{\epsilon} \hat{x}
$$

where $F_{\epsilon}$ is designed by a low-gain method as described in [12]. As shown in [12], since all eigenvalues of $\tilde{A}$ are in the closed left-half plane, $F_{\epsilon}$ can be chosen arbitrarily small; thus, it can be designed so that $\left\|u_{3}(t)\right\| \leq 1 \forall t$.

In this simple example, the values of $k_{1}$ and $k_{2}$ were selected (by trial and error) to be 0.1 , and the low-gain parameter, $\epsilon$, was selected to be 0.001 . The resulting control gain matrix was $F_{\epsilon}=\left(\begin{array}{lll}0.0316 & 0.1010 & 0.1456\end{array}\right)$. The system was simulated, and the resulting state trajectory, state estimate trajectory, and inputs were plotted. (See Fig. 1 and 2.)

The method for designing the controller at station three that guarantees that $u_{3}$ does not saturate is well documented and is basically reduced to tuning a single parameter, $\epsilon$. 


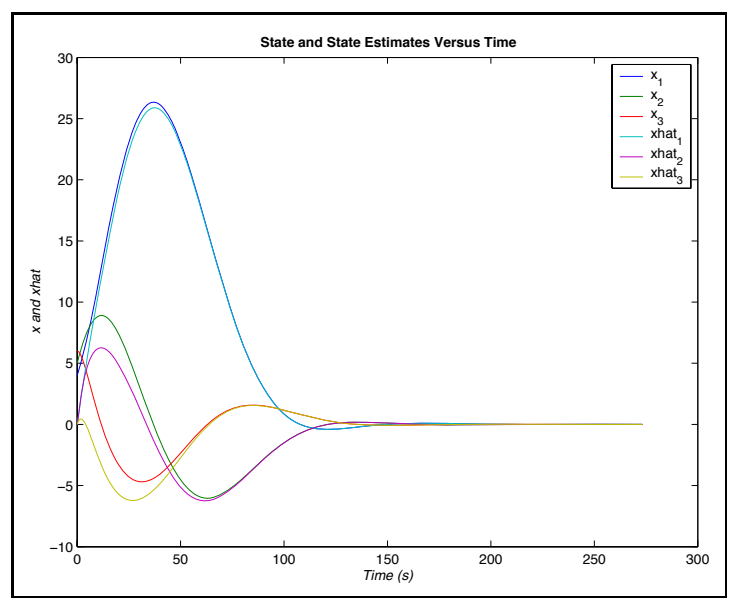

Fig. 1. Simulated State and State Estimate Trajectories.

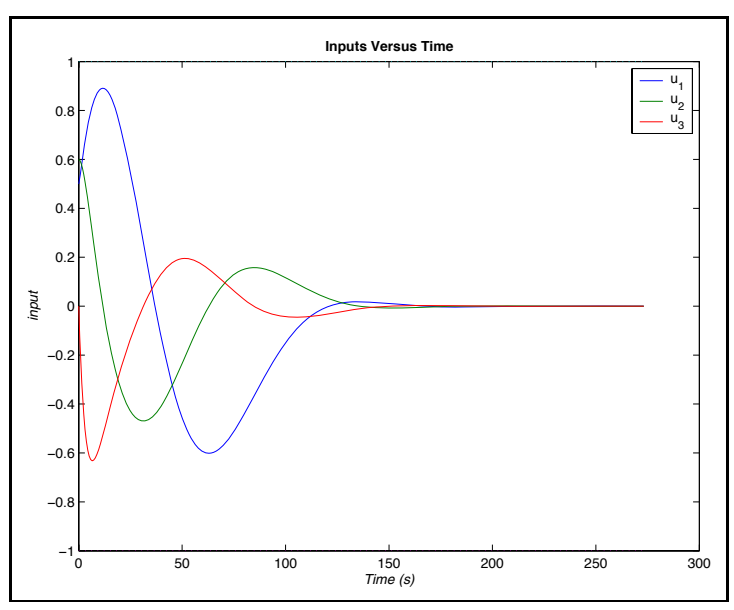

Fig. 2. Simulated Inputs.

However, guaranteeing that the other two inputs remain linear is not as straightforward. Simply making the two preliminary feedbacks smaller does not necessarily make $u_{1}$ and $u_{2}$ smaller, since the magnitude of these feedbacks affects the overshoot in the state itself. Therefore a special design technique is needed in order to guarantee semi-global stability.

A first step towards this goal is decoupling the preliminary and observer-based feedbacks by choosing the same feedback parameter for the first two channels $k_{1}=k_{2}=\alpha$ and making the substitutions $\varepsilon=\alpha \bar{\varepsilon}, F_{\varepsilon}=\alpha \bar{F}_{\bar{\varepsilon}}$. The new gain $\bar{F}_{\bar{\varepsilon}}$ has the same properties as $F_{\varepsilon}$, but it is independent of $\alpha$. Assuming linearity is preserved, the closed-loop will be

$$
\dot{\tilde{x}}=A_{\mathrm{cl}} \tilde{x}
$$

where

$$
\tilde{x}=\left(\begin{array}{c}
x \\
\delta
\end{array}\right), A_{\mathrm{cl}}=\alpha\left(\begin{array}{cc}
\bar{A}-B_{3} \bar{F}_{\bar{\varepsilon}} & B_{3} \bar{F}_{\bar{\varepsilon}} \\
0 & \bar{A}-\bar{L} C_{3}
\end{array}\right),
$$

$\tilde{A}=\alpha \bar{A}$ and $L=\alpha \bar{L}$. The factorization of the preliminary feedback parameter $\alpha$ will provide more freedom in choosing appropriate values for the other parameters such that the inputs do not saturate.

The possible non-linearities caused by the overshoot in the error dynamics can be avoided by splitting the design into two phases: first we make use of the saturation elements on the third channel in order to train the observer, while in the second phase appropriately chosen parameters will keep the inputs in the linear domain once the observer error is small enough. It can be proven that for any $\mu>0$ and any $T>0$, there exists a gain $L$ such that

$$
\|\delta(t)\| \leq e^{-\mu t}\|\delta(0)\| \forall t \geq T .
$$

For the first phase $(0 \leq t \leq T), T$ and $\alpha$ should be chosen small enough such that the state remains inside a given compact set, and the preliminary feedbacks do not saturate (in order to preserve the linearity of the observer error dynamics), while in the second phase ( $t \geq T$ ), using (11) and the decoupling between $\alpha$ and $\bar{\varepsilon}$, appropriately chosen $\alpha, \mu$ and $\bar{\varepsilon}$ will keep all inputs in the linear domain.

\section{Model Reduction by Singular Perturbation}

To date, the literature contains very few methods for designing controllers for decentralized systems, especially in combination with an additional constraint such as input saturation. One tool that may potentially aid in developing such a design method is model reduction by singular perturbation. In this section, we briefly summarize the steps involved in model reduction by singular perturbation and discuss both the potential simplification and the drawbacks of using such a reduced-order model for controller design. (Extensive literature about singular perturbation methods exists, see for instance [13]).

In centralized linear systems with input saturation, when considering stabilization problems, the stable dynamics can be ignored. This reduces the complexity of design considerably since the unstable dynamics are often of very low dimension. In decentralized control, this cannot be done because the stable dynamics are often crucial for the communication between the different channels.

In this paper, we look at systems whose unstable modes are only at the origin. We will show by singular perturbation methods that we can actually achieve a reduction in which we only need to investigate the unstable dynamics. Actually, this reduction reduces the dynamic interconnections induced by the stable dynamics to static interconnections which obviously will allow for an easier design. But this method is obviously only a first step since we do not present a full design for stabilization after we achieve the reduction.

The singular perturbation method can be applied to systems whose dynamics can clearly be divided into fast and slow dynamics. The singular perturbation model for an LTI system is

$$
\Sigma_{S P}:=\left\{\begin{array}{l}
\dot{x}_{1}=A_{11} x_{1}+A_{12} x_{2}+B_{1} u \\
\epsilon \dot{x}_{2}=A_{21} x_{1}+A_{22} x_{2}+B_{2} u \\
y=C_{1} x_{1}+C_{2} x_{2}
\end{array}\right.
$$


where the first and second lines of (12) describe the slow and fast dynamics of the system, respectively. The parameter $\epsilon$ is a measure of the magnitude of the ratio between the large and small eigenvalues.

A zeroth-order approximation is obtained by setting $\epsilon=$ 0 in (12), which is equivalent to assuming that the fast dynamics converge instantly to what is called a quasi-steady state. Thus, the reduced-order model becomes

$$
\Sigma_{R O}:=\left\{\begin{array}{l}
\dot{\bar{x}}_{1}=A_{0} \bar{x}_{1}+B_{0} u \\
y=C_{0} \bar{x}_{1}+D_{0} u,
\end{array}\right.
$$

where $A_{0}=A_{11}-A_{12} A_{22}^{-1} A_{21}, B_{0}=B_{1}-A_{12} A_{22}^{-1} B_{2}$, $C_{0}=C_{1}-C_{2} A_{22}^{-1} A_{21}$, and $D_{0}=-C_{2} A_{22}^{-1} B_{2}$.

A question that arises is how far this reduced-order model is from the actual model. Since the initial value of the approximation for $x_{2}$ (that is, its quasi-steady-state value) may not be close to the actual initial state, the best we can expect is that after a time $t_{1}$, the approximation $\bar{x}$ is close enough to the actual $x_{2}$ :

$$
x_{2}(t)=\bar{x}_{2}(t)+O(\epsilon) \quad \forall t \geq t_{1} .
$$

We do not have the same problem with the approximation $\bar{x}_{1}$, for which

$$
x_{1}(t)=\bar{x}_{1}(t)+O(\epsilon) \quad \forall t \geq 0 .
$$

We now state a few results regarding $\Sigma_{R O}$ and relating to the stability of the $\Sigma_{R O}$ to $\Sigma_{S P}$.

By Tikhonov's Theorem (see for instance [13]), we have the following result:

Theorem V.1 Assume the fast dynamics are asymptotically stable; i.e. , the eigenvalues of the matrix $A_{22}$ are in the open left-half plane. Then (15) holds for all $t \in\left[t_{0}, \infty\right)$, and there exists a $t_{1} \geq t_{0}$ such that (14) holds.

Moreover a static feedback $u=f\left(\bar{x}_{1}\right)$ that asymptotically stabilizes (13) results in a static feedback $u=f\left(x_{1}\right)$ that asymptotically stabilizes (12).

We will now outline the basic steps for model reduction for a stabilizable decentralized system with input saturation (that is, a system with all eigenvalues in the closed left-half plane and no fixed modes on the imaginary axis). Via a basis transformation, such a system can be written as:

$$
\begin{aligned}
\left(\begin{array}{c}
\dot{x}_{1}(t) \\
\dot{x}_{2}(t)
\end{array}\right) & =\left(\begin{array}{cc}
A^{1} & 0 \\
0 & A^{2}
\end{array}\right)\left(\begin{array}{l}
x_{1}(t) \\
x_{2}(t)
\end{array}\right)+\left(\begin{array}{l}
B^{1} \\
B^{2}
\end{array}\right) u(t) \\
y(t) & =\left(\begin{array}{ll}
C^{1} & C^{2}
\end{array}\right)\left(\begin{array}{l}
x_{1}(t) \\
x_{2}(t)
\end{array}\right),
\end{aligned}
$$

where all eigenvalues of $A^{1}$ are zero, $A^{1}$ is in Jordan form, and all eigenvalues of $A^{2}$ are in the open left-half plane. Also, $B$ and $C$ are the partitioned concatenations of all of the $B_{i}$ 's and $C_{i}$ 's, respectively. We also point out that such a basis transformation does not invalidate the decentralized structure, since a basis transformation does not change the input-output behavior.
Applying the change of variable $\tau=\epsilon t$ to (16) gives

$$
\begin{aligned}
\dot{x}_{1}(\tau) & =\frac{1}{\epsilon} A^{1} x_{1}(\tau)+\frac{1}{\epsilon} B^{1} u(\tau) \\
\epsilon \dot{x}_{2}(\tau) & =A^{2} x_{2}(\tau)+B^{2} u(\tau) .
\end{aligned}
$$

Since (18) cannot be evaluated at $\epsilon=0$, we apply the following state transformation.

$$
\left(\begin{array}{l}
\tilde{x}_{1}(\tau) \\
\tilde{x}_{2}(\tau)
\end{array}\right)=\left(\begin{array}{cc}
T_{\epsilon} & 0 \\
0 & I
\end{array}\right)\left(\begin{array}{l}
x_{1}(\tau) \\
x_{2}(\tau)
\end{array}\right)
$$

Recall that all eigenvalues of $A_{1}$ are zero, and $A_{1}$ is in Jordan form; that is, $A_{1}=\operatorname{diag}\left(\begin{array}{lll}J_{1} & \cdots & J_{k}\end{array}\right)$, where each Jordan block, $J_{i}$, has dimension $n_{i}$. Thus, if the transformation matrix is

$$
T_{\epsilon}=\left(\begin{array}{cccc}
T_{1, \epsilon} & 0 & \cdots & 0 \\
0 & T_{2, \epsilon} & \ddots & \vdots \\
\vdots & \ddots & \ddots & 0 \\
0 & \cdots & 0 & T_{k, \epsilon}
\end{array}\right)
$$

where

$$
T_{i, \epsilon}=\left(\begin{array}{cccc}
\epsilon^{n_{i}} & 0 & \cdots & 0 \\
0 & \epsilon^{n_{i}-1} & \ddots & \vdots \\
\vdots & \ddots & \ddots & 0 \\
0 & \cdots & 0 & \epsilon
\end{array}\right) ;
$$

for $i=1, \ldots, k$, then the transformed system fits the singular perturbation model:

$$
\begin{gathered}
\dot{\tilde{x}}_{1}(\tau)=A_{\epsilon}^{1} \tilde{x}_{1}(\tau)+B_{\epsilon}^{1} u(\tau) \\
\epsilon \dot{\tilde{x}}_{2}(\tau)=A_{\epsilon}^{2} \tilde{x}_{2}(\tau)+B_{\epsilon}^{2} u(\tau) \\
y(\tau)=C_{\epsilon}^{1} \tilde{x}_{1}(\tau)+C_{\epsilon}^{2} \tilde{x}_{2}(\tau),
\end{gathered}
$$

where $A_{\epsilon}^{1}=\frac{1}{\epsilon} T_{\epsilon}^{-1} A^{1} T_{\epsilon}=A^{1}$ (because of the structure of $\left.A^{1}\right) ; A_{\epsilon}^{2}=A^{2} ; B_{\epsilon}^{1}=\frac{1}{\epsilon} T_{\epsilon} B^{1} ; B_{\epsilon}^{2}=B^{2} ; C_{\epsilon}^{1}=C^{1} T_{\epsilon}^{-1}$; $C_{\epsilon}^{2}=C^{2} ;$ and $\tilde{x}_{2}(\tau)=x_{2}(\tau)$.

The only problem with this transformed system is that $y(\tau)$ is undefined for $\epsilon=0$ (since $C_{\epsilon}^{1}=C^{1} T_{\epsilon}^{-1}$, and $T_{\epsilon}^{-1}$ is a diagonal matrix with powers of $\epsilon^{-1}$ on the diagonal). However, if we scale each output by $\epsilon^{k_{i}}$, where $k_{i}$ is the largest power of $\epsilon^{-1}$ on the $i^{t h}$ row of $C_{\epsilon}^{1}$, then the new, scaled output,

$$
\tilde{y}(\tau)=\tilde{C}_{\epsilon}^{1} \tilde{x}_{1}(\tau)+\tilde{C}_{\epsilon}^{2} \tilde{x}_{2}(\tau),
$$

is defined for all finite $\epsilon$. Such scaling does not affect the structure of our system. From (21), (22), and (24), we find the following singular perturbation model for our system:

$$
\Sigma_{\epsilon}:= \begin{cases}\dot{\tilde{x}}_{1}(\tau) & =A^{1} \tilde{x}_{1}(\tau)+B_{\epsilon}^{1} u(\tau) \\ \epsilon \dot{\tilde{x}}_{2}(\tau) & =A^{2} \tilde{x}_{2}(\tau)+B^{2} u(\tau) \\ \tilde{y}(\tau) & =\tilde{C}_{\epsilon}^{1} \tilde{x}_{1}(\tau)+\tilde{C}_{\epsilon}^{2} \tilde{x}_{2}(\tau)\end{cases}
$$

The fast dynamics are asymptotically stable, while the slow dynamics have all eigenvalues at the origin. When considering the slow dynamics, we first note that the 
decentralized structure is preserved. By setting $\epsilon=0$ in (25), we actually obtain a system of the form:

$$
\Sigma_{0}:=\left\{\begin{array}{l}
\dot{\bar{x}}=\bar{A} \bar{x}+\sum_{i=1}^{k} \bar{B}_{i} u_{i} \\
y_{i}=\bar{C}_{i} \bar{x}+\sum_{j=1}^{k} \bar{D}_{i j} u_{j}, \quad i=1, \ldots, k .
\end{array}\right.
$$

We first note that the slow dynamics are always centralized stabilizable. The system in general need not be centralized detectable. However, in important cases such as when all Jordan blocks associated with the eigenvalue 0 have the same size, then the slow dynamics are centralized detectable.

Another important issue is whether reduction to the slow dynamics can introduce fixed modes at the origin that were not present in the original system. Again, this is not the case when all Jordan blocks associated with the zero eigenvalue have the same size. The same conclusion holds also if, instead of assuming that all the Jordan blocks in $\bar{A}$ have the same size, we assume that observations of different Jordan blocks do not interact (i.e. they appear on different rows in $\bar{C}$ ). Also notice that under either of these two assumptions, the centralized detectability of the reduced system is guaranteed as well. However, detectability of the reduced system does not necessarily imply absence of fixed modes at the origin.

For our stabilization purposes, a more specific version of Theorem V.1 holds in this case, allowing also dynamic stabilizing controllers for the reduced system:

\section{Theorem V.2 If a dynamic controller}

$$
C:\left\{\begin{array}{l}
\dot{v}=G_{1} v+G_{2} y \\
u=F_{1} v+F_{2} y
\end{array}\right.
$$

stabilizes the reduced system $\Sigma_{0}$, and $A^{2}+B^{2} F_{2} \tilde{C}_{0}^{2}$ is asymptotically stable, then there exists $\epsilon^{*}>0$ such that $C$ also stabilizes $\Sigma_{\epsilon}$ for all $\epsilon \in\left(0, \epsilon^{*}\right)$.

This result is valid for general (centralized) controllers, so it will hold also for a particular decentralized controller that does not saturate and asymptotically stabilizes the reduced system. Regarding the design, it is interesting to note that the interconnections between the different channels have become mostly static and the only possible remaining dynamics are integrators. Therefore, the crucial aspect in stabilization has become the graph structure underlying the interconnections, which directly connects to other recent approaches such as the authors in [14] recently proposed in designing controllers for decentralized systems in which the dynamics are simple but the interconnection structure is arbitrarily complex.

\section{CONCLUSION}

In this article, we have explored some of the inherent difficulties in designing stabilizing controllers for decentralized control systems with input saturation. We have shown that (at least under certain conditions) time-invariant nonlinear controllers cannot be used to move fixed modes at zero. We also provided an example to illustrate the potential problems caused by peaking in an observer-based design. This phenomenon certainly makes designing a linear stabilizing controller more difficult but not impossible, and we outlined a procedure for designing such a controller. Finally, we adapted the theory for model reduction by singular perturbation to decentralized systems, in which the stable dynamics cannot be ignored because they play a crucial role in communication between control stations. We feel that this model reduction method is a very promising first step in designing stabilizing controllers for decentralized control systems with input saturation.

\section{REFERENCES}

[1] S. Wang and E. Davison, "On the stabilization of decentralized control systems," IEEE Trans. Aut. Contr., vol. 18, no. 5, pp. 473478, 1973.

[2] J. Corfmat and A. Morse, "Decentralized control of linear multivariable systems," Automatica, vol. 12, no. 5, pp. 479-495, 1976.

[3] B. Anderson and D. Clements, "Algebraic characterization of fixed modes in decentralized control," Automatica, vol. 17, no. 5, pp. 703$712,1981$.

[4] B. Anderson and J. Moore, "Time-varying feedback laws for decentralized control," IEEE Trans. Aut. Contr., vol. 26, no. 5, pp. 11331139, 1981.

[5] Z. Gong and M. Aldeen, "Stabilization of decentralized control systems," J. Math. Syst. Estim. Control, vol. 7, no. 1, pp. 1-16, 1997.

[6] J. Willems, "Time-varying feedback for the stabilization of fixed modes in decentralized control systems," Automatica, vol. 25, no. 1, pp. 127-131, 1989.

[7] P. Khargonekar and A. Özgüler, "Decentralized control and periodic feedback," IEEE Trans. Aut. Contr., vol. 39, no. 4, pp. 877-882, 1994.

[8] A. Fuller, "Linear control of non-linear systems," Int. J. Contr., vol. 5, no. 3, pp. 197-243, 1967.

[9] E. Sontag and H. Sussmann, "Nonlinear output feedback design for linear systems with saturating controls," in Proc. 29th CDC, Honolulu, 1990, pp. 3414-3416.

[10] Z. Gong and M. Aldeen, "On the characterization of fixed modes in decentralized control," IEEE Trans. Aut. Contr., vol. 37, no. 7, pp. 1046-1050, 1992.

[11] Z. Lin and A. Saberi, "Semi-global exponential stabilization of linear systems subject to "input saturation" via linear feedbacks," Syst. \& Contr. Letters, vol. 21, no. 3, pp. 225-239, 1993.

[12] A. Saberi, Z. Lin, and A. Teel, "Control of linear systems with saturating actuators," IEEE Trans. Aut. Contr., vol. 41, no. 3, pp. 368-378, 1996.

[13] P. Kokotović, H. Khalil, and J. O'Reilly, Singular perturbation methods in control: analysis and design, ser. Classics in Applied Mathematics. Philadelphia: SIAM, 1999, vol. 25.

[14] S. Roy, A. Saberi, and K. Herlugson, "Formation and alignment of distributed sensing agents with double-integrator dynamics," 2004, accepted for publication in the IEEE Press monograph "Sensor Network Operations". 